\title{
Fragmentation of grains in a two-dimensional packing ${ }^{\star}$
}

\author{
J.A. Åström ${ }^{1, a}$ and H.J. Herrmann ${ }^{1,2}$ \\ ${ }^{1}$ Laboratoire de Physique Mécanique des Milieux Hétérogènes, École Superieure de Physique et Chimie \\ Industrielles, 10 rue Vauquelin, 75231 Paris, Cedex 05, France \\ 2 ICA1, University of Stuttgart, Pfaffenwaldring 27, 70569 Stuttgart, Germany
}

Received: 14 December 1997 / Accepted: 17 March 1998

\begin{abstract}
A numerical model of fragmentation of a two-dimensional granular medium under pressure is investigated. The fragmentation process is found to be strongly dependent on the type of force used as the criterion for breaking the grains. The fragmentation mode affects the process less dramatically. There is a power-law divergence in the pressure when the medium approaches the full packing limit, $\left(\epsilon_{c}-\epsilon\right)^{-2.0}$. Both log-normal and power-law fragment-size distributions are found. Gravity is demonstrated to be an important factor.
\end{abstract}

PACS. 83.70.Fn Granular solids - 46.30.Nz Fracture mechanics, fatigue, and cracks - 02.70.Ns Molecular dynamics and particle methods

\section{Introduction}

The process of fragmentation of a set of particles into smaller ones by pressing them against each other is of vast technological importance $[1,2]$. Also many natural processes ranging in size from the collisional evolution of asteroids to the formation of dust, belong, at least to some degree, to this category. Consequently, there have been many investigations devoted to this subject (good reviews are given in Refs. [2-4]). Much interest has been focused on understanding the origin of the fragment-size distribution [2-13]. Both log-normal and power-law distributions appear commonly in experimental and theoretical studies. The origin of the log-normal size distribution is quite easy to understand. If all grains and fragments break always independently of each other and with a more or less constant probability per unit time, then, after a long time, the number of breakings per unit mass will have a normal distribution. If at each breaking, $a$ fragments are formed, then the number, $c(i)$, of fragments that have gone through $i$ breakings is given by

$$
c(i)=\frac{N_{0} a^{i}}{\sqrt{2 \pi}} \exp \left[-(i-\bar{i})^{2} / 2\right],
$$

where $N_{0}$ is the original number of particles, and $\bar{i}$ the average number of breakings per mass-unit. The reduction in the particle radius will typically be $r_{i}=a^{-i / 2} r_{0}$. By combining this with equation (1) a log-normal distribution is obtained. In nature, log-normal size distributions are found in both boulder fields and soils [14].

\footnotetext{
${ }^{\star}$ Dedicated to J. Zittartz on the occasion of his 60th birthday

a e-mail: astrom@cypress.csc.fi
}

The other commonly appearing type of size distribution is the power-law distribution. An interesting feature of power-law fragment size distributions is that it is possible to fill the entire space with fragments even if they have a 'problematic' shape like circles. This can be done by choosing the right exponent and arranging the fragments on a fractal set [15]. For circles in two dimensions the exponent of one possible solution is close to -2.3 (i.e. $n(r) \propto r^{-2 \tau}$, where $2 \tau=d_{f}+1$ and $d_{f} \approx 1.3$ is the fractal dimension of Apollonian packing). It has also been suggested that a power-law size distribution is a result of self-organized criticality [5]. In experiments, power-law distributions commonly result from highly energetic impacts such as explosions [2], but also ongoing fragmentation can lead to a power-law size distribution [3].

In this paper we investigate a numerical twodimensional model for the fragmentation of a granular medium under pressure. We model the grains and fragments as elastic circular discs. At the begining of a simulation a few grains are placed at random in a twodimensional box. If the grains overlap they repell each other with a force $F=E\left(r_{i}+r_{j}-\delta s\right)^{1.5} / \sqrt{1 / r_{i}+1 / r_{j}}$ [16], where $r_{i}, r_{j}$ are the radii of the overlapping grains, $\delta s$ the distance between the centres of the grains, and $E$ Young's modulus. The walls of the box are also elastic. The pressure is increased by decreasing the size of the box. The grains move according to Newton's equations of motion. Energy is being dissipated by introducing an extra, 'viscous' dissipation term in the equations of motion [17]. That is, a force proportional to the velocity is slowing down a particle.

Choosing the rules according to which the grains break is far from trivial. First, the type of load that causes 


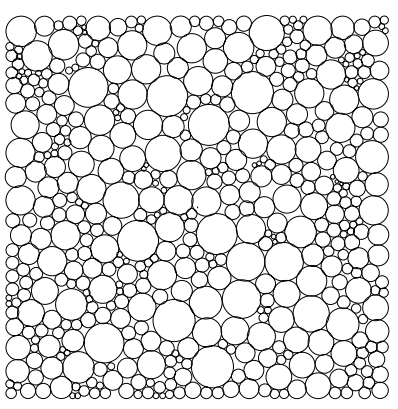

(a)

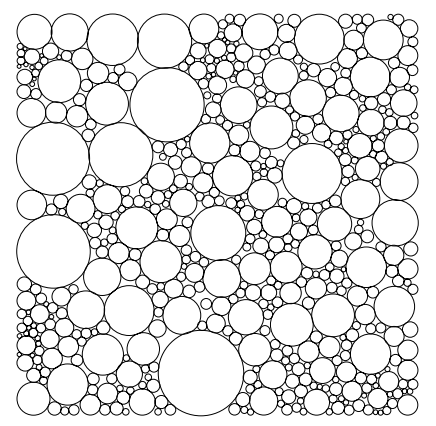

(b)
Fig. 1. Two fragmented systems containing roughly 500 fragments. (a) Breaking rule (B) and fracture mode (I). (b) Breaking rule (B) and fracture mode (II). $E=5000, N_{0}=50$, $r \in[0.25,0.75], M \approx 40$.

fragmentation has to be set. In this paper we report simulation results with two different types of fragmentation criteria; (A) a threshold value for the pressure on a grain, and (B) a threshold value for the largest compressive force at the contacts of a grain. The first type of load is a quite natural choice as the pressure is the total compressive force on a grain. The second type is more delicate. If the grains are made of a stiff material, and their surfaces are smooth, then the contacts between grains will be almost pointlike. This leads to a high stress concentration at these points. For a brittle material it is known that high stress concentrations are the origin of crack propagation [18], which leads to fragmentation.

The second rule that has to be set is the fracture mode, i.e. how an individual particle fragments. Obviously, the total mass has to be conserved during fragmentation. In order to handle the fragments in a similar way as the grains we want to model also all fragments as circular discs. The problem with choosing a good fracture mode is then that; (1) the number of fragments at each breaking should be kept low so that many breakings can be made in each simulation, (2) the fragments should be chosen so that they can be packed in such a way that local pressure decreases at a breaking, and (3) the breaking mechanism should at least to some extent mimic the real event. To simultaneously satisfy all these three critera is obviously impossible. Instead of doing a compromise, however, we use two different criterions. In the first (I) we simply split a grain in two equal-size fragments. This criterion violates (2) and induces strong, unphysical local pressures. In the second (II) we pack 12 fragments of three different sizes into the area of the broken grain and place the rest of the mass just outside in the 'pockets' between the surrounding grains. This criterion violates (1), but in most cases satifies (2) (the local preasure can occasionaly increase a little for a short time just after a breaking if the fragments do not really fit in the pockets). Criterion (3) is also reasonably well satisfied.

The results of two simulation runs, starting with 50 grains with radii uniformly distributed between 0.25 and 0.75 , and fragmenting up to 500 particles are shown in
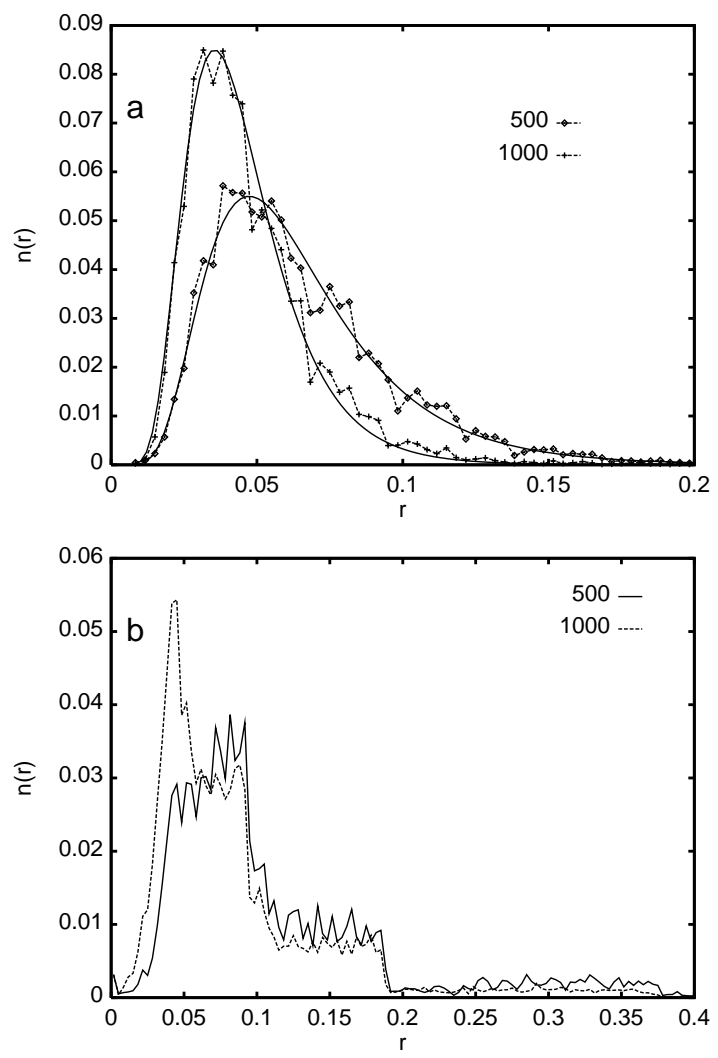

Fig. 2. Fragment size distributions of the systems obtained with mode (I) and mode (II), respectively. Results are shown for 500 and 1000 fragments. Log-normal distributions are fitted for mode $(\mathrm{I}) \cdot n(r)=0.055 \exp \left[-(\ln (r)+3.05)^{2} / 0.43\right]$ for 500 fragments and $n(r)=0.085 \exp \left[-(\ln (r)+3.34)^{2} / 0.33\right]$ for 1000 fragments.

Figure 1. Breaking rule (B) was used, and Figures 1a and $1 \mathrm{~b}$ are results of fracture modes (I) and (II), respectively. The most striking difference between the two modes is that some grains remain unbroken using mode (II) (the largest circles in Fig. 1b). This effect is rather easy to understand. Using mode (II) the radii are decreasing fast, which means that small fragments will surround unbroken grains. When this happens the force on a unbroken grain will be distributed among several contacts, which means that none of them will be large enough to break the grain. In spite of this, the fragment size distributions resulting from Figures $1 \mathrm{a}$ and $1 \mathrm{~b}$ (averaged over many simulation runs though) does not differ very much from each other. The general shape of both distributions is lognormal (Fig. 2) and the mean fragment sizes decrease roughly in a similar fashion for the two modes. Using mode (I) and starting with 50 grains with a mean size of 0.5 results in mean sizes 0.14 and 0.10 for 500 and 1000 fragments, respectively. For mode (II) the mean sizes are 0.12 and 0.09 .

To investigate the development of the 'hydrodynamic' pressure $(P)$ in the system, we define strain as $\epsilon=\left(L_{0}-\right.$ $L(t)) / L_{0}$, where $L_{0}$ is the original length of the simulation box, and $L(t)$ the time-dependent length. Let us define $\epsilon_{c}$ 

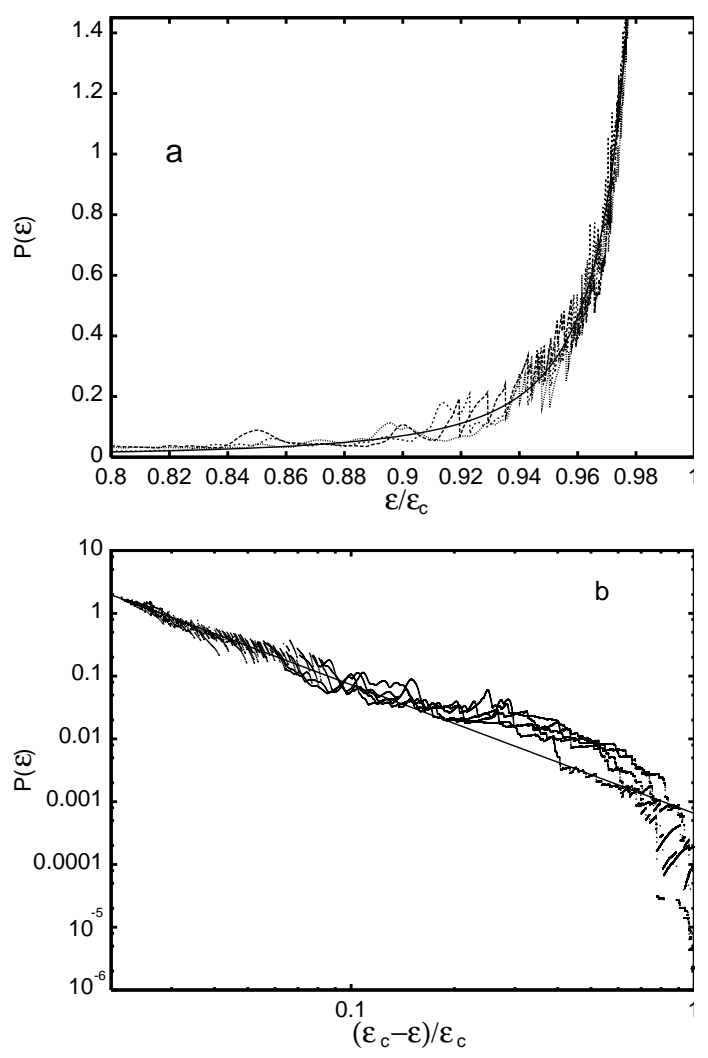

Fig. 3. The pressure $P$ as a function of $\epsilon$ for systems similar to the one in Figure 1b. Individual breakings of grains appear as sharp pressure drops. For comparison a function proportional to $\left(1-\epsilon / \epsilon_{c}\right)^{-2}$ is also plotted. (a), (b) Linear and logarithmic scale, respectively.

as $\epsilon_{c}=\left(L_{0}-\sqrt{M}\right) / L_{0}$, where $M$ is the total surface area (or mass) of the grains and fragments. $P(t)$ is shown as a function of $\epsilon(t) / \epsilon_{c}$ in Figure 3 for criterion (B) and mode (II). At the end of these simulations (i.e. at $\epsilon / \epsilon_{c} \approx$ $0.98)$ there are about $8-9 \%$ of voids and the 'overlap' of circles is less than $0.5 \%$. The pressure seems to diverge as $\epsilon$ approaches $\epsilon_{c}$, although there is a cutoff as the particles are elastic. This divergence is approximately of the form $\left(1-\epsilon / \epsilon_{c}\right)^{-2}$ (full lines in Fig. 3). The origin of the value of this exponent is, however, not known to us.

Up to this point we have not investigated breaking criterion (A). Fragmentation with this criterion is dominated by an 'instability' in the sense that smaller particles break easier than large ones. An indication of this instability was found by investigating the pressure (without breaking) as a function of grain size [19]. For a bidisperse grain size distribution it was found that the average pressure does depend on the radius of a grain, but the high pressure tail of the distribution was found to be completely dominated by small grains. Thus we expect that once fragmentation is initiated at one spot it will continue breaking the ever smaller fragments at that location. This is indeed what is found in the simulations with both modes (I) and (II) (Fig. 4). The fragment size distribution can easily be estimated in this process. If each breaking results in $a$ fragments of equal size, then the radius of a fragment after $i$
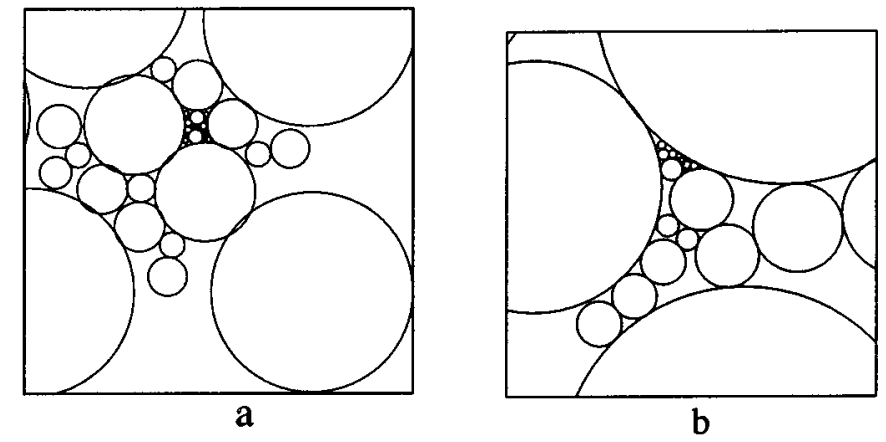

Fig. 4. Detailed picture of the breaking locations in two systems using breaking rule (A). (a) Fracture mode (II) and (b) fracture mode (I). No fracture occured outside the pictures.

breakings will, as above, be $r_{i}=r_{0} a^{-i / 2}$. If we now assume that only the smallest fragments can break (there can, however, be many with the same size), and that a fraction $p$ of them will break, then the number of fragments resulting from the $i$ :th breaking will be $(1-p) N_{0}(p a)^{i}$. Using relation between $r_{i}$ and $i$ (given above), the number of fragments with radius between $r$ and $r+\delta r$ can be obtained. The result is

$$
n(r)=\frac{(1-p) N_{0} \sqrt{a}}{r_{0}(\sqrt{a}-1)}\left(r / r_{0}\right)^{-[2 \ln (p) / \ln (a)+3]} \delta r .
$$

If, strictly, only the smallest fragment (i.e. just one) can break, then only one fragment out of $a$ can continue fragmenting. This means that $p=1 / a$, which leads to $n(r) \propto 1 / r$. If, on the other hand, almost all fragments continue to break (i.e. $p \rightarrow 1$ ), then $n(r)$ will be proportional to $r^{-3}$. The exponent decreases continuosly from -1 to -3 as $p$ increases from $1 / a$ to 1 .

In order to test equation (2) we did simulations starting with 10 fragments of nearly equal size and runing up to 150 fragments using both modes (I) and (II). The instability occured in both cases. In Figure 4 we show detailed pictures of the breaking locations for the two modes. No fragmentation occured outside the figure areas, and the resulting fragment size distributions were of the form $1 / r$ in both cases. In order to find other exponents we tried varying different parameters (like the stiffness of the grains, the strain rate, and the original size distribution). The same fragments size distribution, $1 / r$, appeared, however, always. We even tried introducing random fragmentation threshold, but no change was seen in the size distribution. It was only with the introduction of gravity that the distribution changed. There is a physical explanation to this; without gravity the small fragments remain more or less at the breaking location, which leads to decreasingly smaller fragments being crushed between large unbroken grains (Fig. 4). In the presence of gravity, however, the small fragments fall between large fragments which leads to rearrangements that affect the final fragment size distribution. In Figure 5 we show a system of 50 grains broken into roughly 800 fragments in the presence of gravity. Also a detailed picture of a fragmentation location is displayed. The contrast to Figure 4 is obvious. 
a
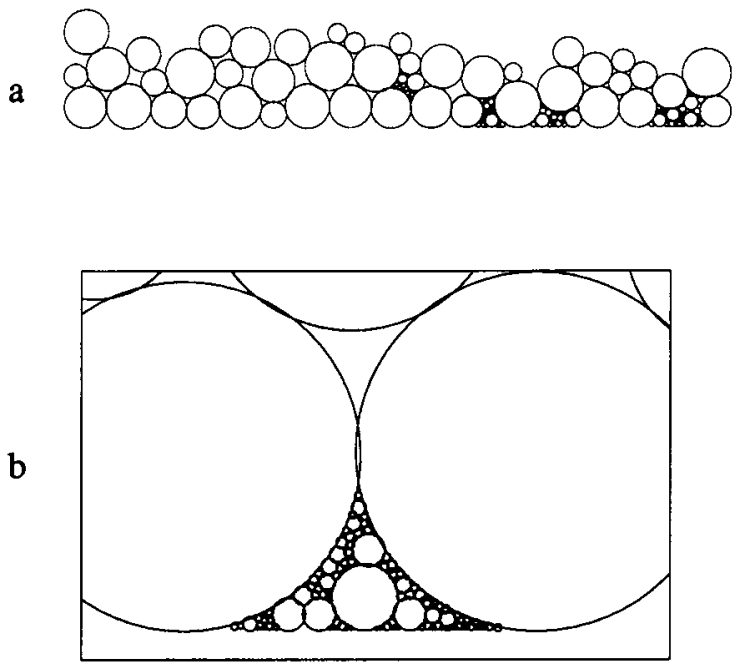

Fig. 5. 800 fragments broken using breaking rule (A) and fracture mode (II) under the influence of gravity. (a) The entire system at the end of a simulation with all grains and fragments located at the bottom of the simulation box. (b) A detailed picture of a breaking location.

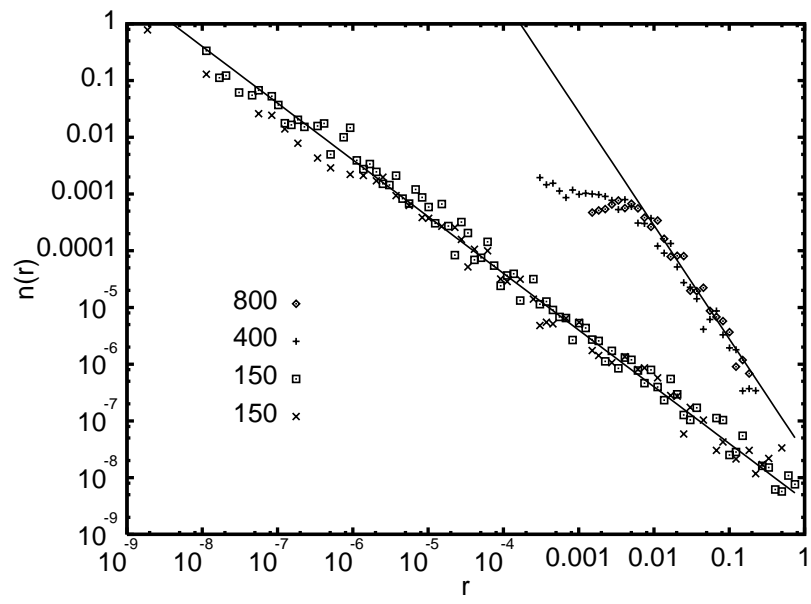

Fig. 6. The fragment size distributions of the systems of Figures 4 and 5 . A function of the form $1 / r$ is fitted in the case of no gravity and a function $1 / r^{2}$ is fitted to the distribution obtained under gravity.

Finally, in Figure 6 we compare the fragment size distributions of Figures 4 and 5 . The fitted lines are functions proportional to $1 / r$ and $1 / r^{2}$, respectively. It is interesting to notice that the fragment size distribution obtained under gravity (i.e. $\left.n(r) \propto 1 / r^{2}\right)$ is fairly close to $n(r) \propto 1 / r^{2.3}$ of an Apollonian packing [15].
In summary, we have demonstrated that the force used in the breaking criterion is crucial to the fragmentation of a granular medium under pressure. With a fracture threshold for the largest contact force, log-normal fragment size distributions are obtained. If the fracture criterion is a threshold value for the pressure on a grain then the process is unstable and results in a size distribution of the form $1 / r$ in the case of no gravity. If gravity is present then rearrangements of particles lead to much more efficient packing and fragment-size distributions similar to those found in Apollonian packing appear.

The authors acknowledge useful discussions with O. Tsoungui, S. Roux and J.C. Charmet.

\section{References}

1. C. Thornton, K.K. Yin, M.J. Adams, J. Phys. D. Appl. Phys. 29, 424 (1996).

2. D.L. Turcotte, J. Geophys. Res. 91, 1921 (1986).

3. S. Redner, in Statistical models for the fracture of disordered media, edited by H.J. Herrmann, S. Roux (NorthHolland, Amsterdam, 1990).

4. S. Redner, in Disorder and Fracture, edited by J.C. Charmet, S. Roux, E. Guyon (Plenum Press, London, 1990).

5. L. Oddershede, P. Dimon, J. Bohr, Phys. Rev. Lett. 71, 3107 (1993).

6. T. Matsui, T. Waza, K. Kani, S. Suzuki, J. Geophys. Res. 87 B13, 10968 (1982).

7. A. Fujiwara, A. Tsukamoto, Icarus 44, 142 (1980).

8. A. Meibom, I. Balslev, Phys. Rev. Lett. 76, 2492 (1996).

9. S. Steacy, C. Sammis, Nature 353, 250 (1991).

10. G. Hernandez, H.J. Herrmann, Physica A 215, 420 (1995).

11. F. Kun, H.J. Herrmann, Int. J. Mod. Phys. C 7, 837 (1996).

12. J. Åström, J. Timonen, Phys. Rev. B 54, R9585 (1996).

13. J. Åström, J. Timonen, Phys. Rev. Lett. 78, 3677 (1997).

14. E.C. Dapples Basic Geology for Science and Engineering, (Wiley, New York, 1959); Models of Geologic Processes, edited by P. Fenner (American Geological Institute, Washington, 1969); P. Habib, Soil and Rock Mechanics (Cambridge University Press, Cambridge, 1983); T.H. Wu, Soil Mechanics (Allyn and Bacon, Boston, 1996).

15. K.J. Falconer, The Geometry of Fractal Sets (Cambridge University Press, Cambridge, 1985); H.J. Herrmann, G. Mantica, D. Bessis, Phys. Rev. Lett. 65, 3223 (1990).

16. H. Hertz, J. Reine Angew. Math. 92, 136 (1882).

17. J. Schäfer, S. Dippel, D.E. Wolf, J. Phys. I France 6, 5 (1996).

18. D.R. Clarke, K.T. Faber, J. Phys. Chem. Sol. 48, 1115 (1987); Y.S. Li, P.M. Duxbury, Phys. Rev. B 40, 4889 (1989).

19. O. Tsoungui, D. Vallet, J.-C. Charmet, S. Roux, C.R. Acad. Sci. Paris 325 II b, 457 (1997). 